\title{
Designing tools for oil exploration using nuclear modeling
}

\author{
Marie-Laure Mauborgne ${ }^{1, \text { a }}$, Françoise Allioli ${ }^{1}$, Mauro Manclossi ${ }^{1}$, Luisa Nicoletti ${ }^{1}$, Chris Stoller $^{2}$, and Mike Evans ${ }^{2}$ \\ 1 Schlumberger Riboud Product Center, Clamart, France \\ 2 Schlumberger Houston Formation Evaluation Integration Center, Sugarland, TX, USA
}

\begin{abstract}
When designing nuclear tools for oil exploration, one of the first steps is typically nuclear modeling for concept evaluation and initial characterization. Having an accurate model, including the availability of accurate cross sections, is essential to reduce or avoid time consuming and costly design iterations. During tool response characterization, modeling is benchmarked with experimental data and then used to complement and to expand the database to make it more detailed and inclusive of more measurement environments which are difficult or impossible to reproduce in the laboratory.

We present comparisons of our modeling results obtained using the ENDF/B-VI and ENDF/B-VII cross section data bases, focusing on the response to a few elements found in the tool, borehole and subsurface formation. For neutron-induced inelastic and capture gamma ray spectroscopy, major obstacles may be caused by missing or inaccurate cross sections for essential materials. We show examples of the benchmarking of modeling results against experimental data obtained during tool characterization and discuss observed discrepancies.
\end{abstract}

\section{Introduction}

In the oilfield, exploration of the subsurface is essential to answer questions regarding location, quantity, type and producibility of hydrocarbons. Well logging provides measurements of rock formation characteristics and fluids in their pore space. Nuclear measurements are central to this evaluation [1].

Such measurements may be obtained either by lowering a downhole tool on wireline into the borehole after the well has been drilled or by including nuclear measurements in the drill string in the bottom hole assembly during drilling. Logging-while-drilling (LWD) tools need to survive in this very rough environment and provide reliable results, while being exposed to repeated severe shocks of more than $100 \mathrm{~g}$ and temperatures that may exceed $175^{\circ} \mathrm{C}$. Sensors and sources must be protected by pressure housings able to withstand pressures of 2000 atmospheres or more. Modeling is essential in defining and refining promising tool concepts, and geometries and in making an optimal choice of materials, while respecting the mechanical constraints of the tool construction. Once a prototype of the tool is available, modeling is benchmarked against tool experimental data and then used to refine the data base and expand it to certain measurement environments which are difficult or impossible to reproduce in the lab.

Principal nuclear measurements are formation natural gamma ray, density, neutron porosity, sigma (macroscopic thermal neutron capture cross section of the formation) and spectroscopy of neutron-induced formation gamma rays. Depending on the measurement, this requires modeling of neutron transport, gamma ray generation, gamma ray transport or combinations thereof.

\footnotetext{
a e-mail: MMauborgne@slb.com
}

Our main modeling objective is to predict and reproduce the direct measurement as accurately as possible, so that we can fully leverage the results during tool and algorithm development. Neutron and gamma ray transport are relatively easy to model separately and benchmarking provides very good results. The same is not true for neutron induced gamma ray generation and subsequent gamma ray transport.

Nuclear measurements in the oilfield use radioactive sources of gamma rays and neutrons in addition to pulsed neutron generators (PNGs). Modeling PNG-based tools is more complex, as the use of neutron pulses allows timedependent measurements and requires modeling of time dependent phenomena.

\section{Use of modeling}

Modeling is widely used when designing a tool to help find the best compromise between mechanical constraints—ensuring tool integrity under shock, vibration and temperature-, and measurement quality. This enables optimization of detector and radiation source positioning and selection of materials surrounding them to maximize the signal-to-noise ratio.

When modeling a logging tool with MCNP [2], for example, the first issue encountered is the very low probability of detecting a neutron or gamma ray emitted from the source. For a PNG-based tool, about 10 particles out of 1 million emitted by the source get back to the farthest detector. Therefore, variance reduction is critical for obtaining precise results.

Once a tool has been designed and built, the modeling is benchmarked against laboratory measurements. At this point, modeling is used to expand, complement and refine the experimental database, which is the foundation of response algorithm development. This allows tool 

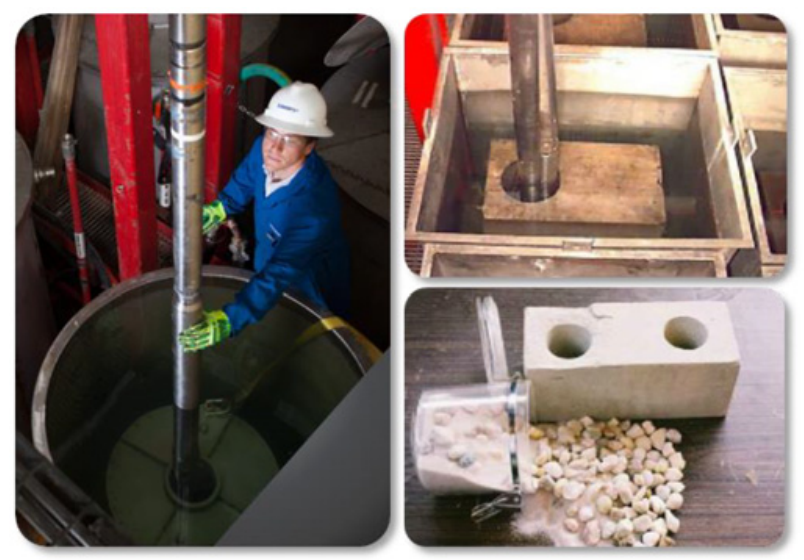

Figure 1. Tools and formation samples for laboratory measurements.

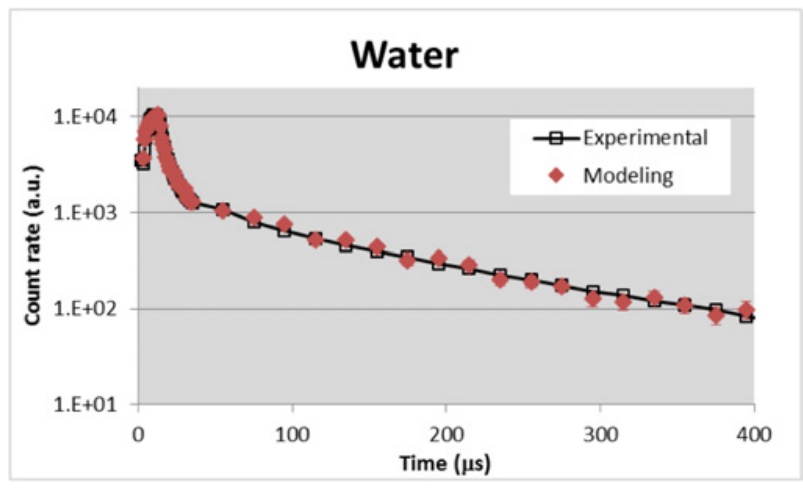

Figure 2. Example of neutron time spectrum in water: comparison between modeling and experimental data.

performance to be predicted more accurately, facilitating the development of better answer products. In the laboratory, only a limited number of actual or simulated rock formations are available. Many formations such as anhydrite or gas-filled formations are, at best, difficult to obtain in a laboratory environment. In addition, for practical and financial reasons, only a small number of rock formations with different properties, such as porosity, lithology, borehole size, and formation fluid are available. This limits the number of laboratory measurements to a few hundred for the large and heavy (up to $3000 \mathrm{~kg}$ ) LWD tools.

Benchmarking of the model is done with laboratory measurements using commercial logging tools. Examples of this are shown in Fig. 1. At the left, a wireline neutron-gamma spectroscopy tool is being prepared for a measurement. The rock is in a small container inside a bigger water tank. The top right shows a gamma-gammadensity measurement in a rock formation. At the bottom right, different rock samples are shown.

Modeling of neutron or gamma-only problems generally gives good results, as in Fig. 2, but coupled neutrongamma problems can exhibit significant discrepancies between measurement and simulation, in particular when dealing with gamma ray production.

Logging measurements introduce certain limitations and challenges, such as the noise (background) signal generated by the tool itself, and limitations on the acquired data, amongst others. In spite of these limitations,

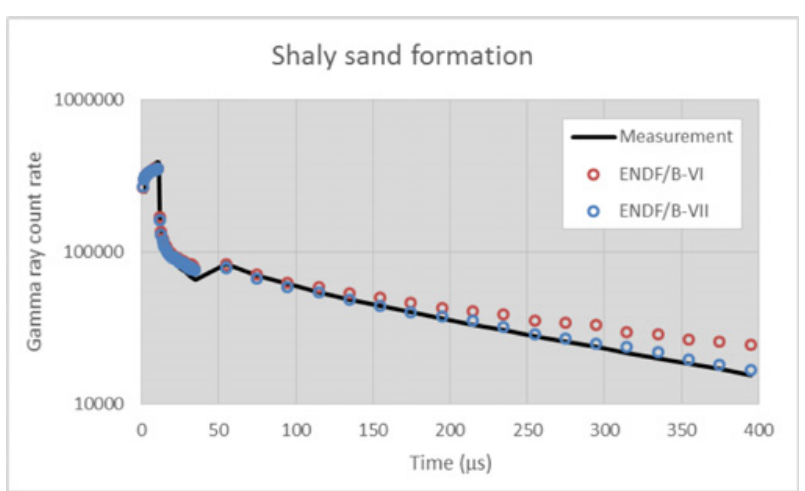

Figure 3. Comparison between modeling results obtained using ENDF/B-VI and ENDF/B-VII libraries for determining the time spectrum in the shaly sand formation.

it has been observed that many discrepancies between measurement and simulation are caused by deficiencies in the modeling and in particular by the lack of accurate gamma ray production cross sections.

\section{Choosing libraries}

The choice of neutron cross section libraries is very important in simulating tool response because the tools themselves often contain elements that are not common in the rest of the nuclear industry. Also, the quality of the required neutron/gamma ray production cross sections is often not sufficiently accurate to faithfully reproduce the details of neutron-induced gamma-ray spectra.

With the introduction of ENDF/B-VII [3], we have seen significant improvements for some elements, but also some degradations compared with ENDF/B-VI [4].

\subsection{Iron}

Iron is often present in shale rock type and, obviously, in our tools. Shale is a sedimentary rock composed of a mix of flakes of clay minerals and tiny fragments of other minerals, especially quartz and calcite. Clay minerals have variable amounts of iron, magnesium, alkali metals, alkaline earths.

We have characterized formations containing iron that we use for benchmarking. One of these simulates a shaly sand. Results from this formation are used to choose the best iron cross section.

Figure 3 shows the gamma ray time spectrum that is measured by the tool after a series of $10 \mu$ s neutron burst. The decay rate determined from this spectrum is used to measure the macroscopic thermal absorption cross section of the formation, called sigma [5]. In turn, sigma is used to identify the type of fluid in the pore space of the formation.

We have performed two different simulations for this formation using the same model, changing only the library for the iron outside the tool. Figure 3 displays the impact on the time decay spectra and Fig. 5 displays the impact on the inelastic and capture gamma ray energy spectra. The best agreement between modeling and experiment is clearly with the use of ENDF/B-VII.

The shape of the time spectrum may look strange because of the discontinuity at $35 \mu \mathrm{s}$. This is due to the way data are recorded. The PNG timing scheme is shown in Fig. 4. The PNG fires 32 times for $10 \mu$ s followed 


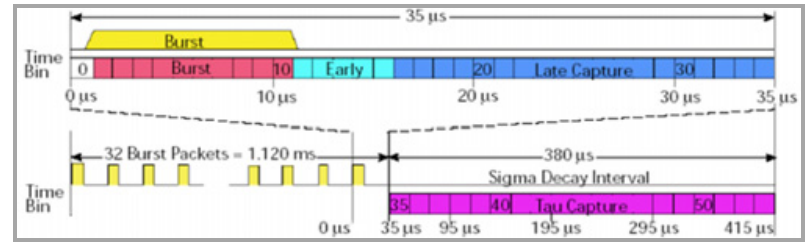

Figure 4. Example of a PNG timing scheme.

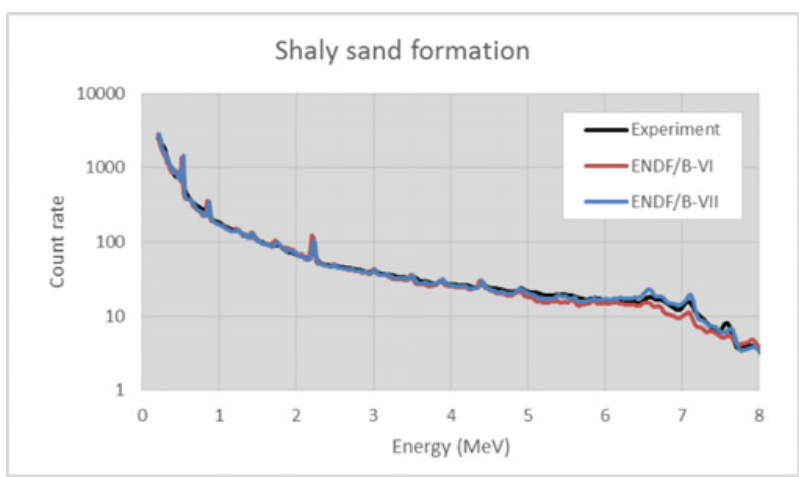

Figure 5. Comparison of ENDF/B-VI and ENDF/B-VII simulated total gamma ray spectra and measurements in the shaly sand formation.

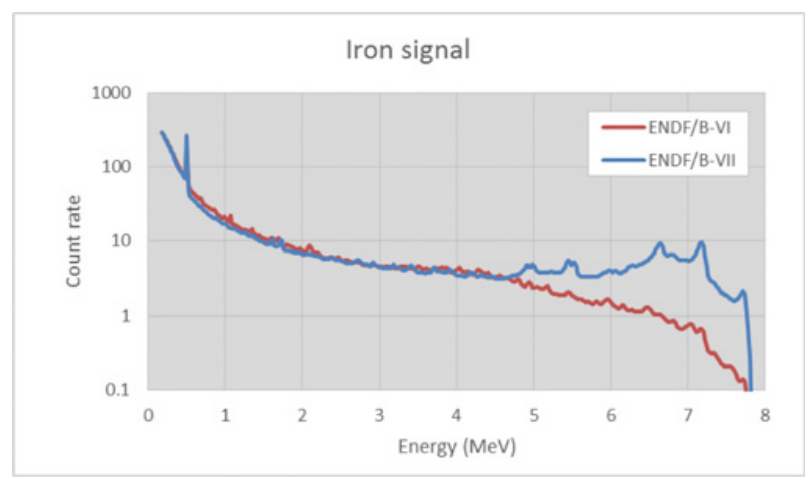

Figure 6. Comparison between ENDF/B-VI and ENDF/B-VII simulated formation iron spectra in the shaly sand formation.

by a $25 \mu$ s pause. Only the average of the 32 packets is recorded. The longer time decay is recorded only after the last of the 32 packets during the longer pause (sigma decay interval). The discontinuity is due to the average of the decays after the 32 neutron bursts being plotted and not the last burst-pause packet.

Figure 5 represents the capture gamma ray energy spectrum that is measured by the tool in the shaly sand formation. The data are used to determine the elemental composition of the formation by spectroscopy. A significant difference at high energy (above $5 \mathrm{MeV}$ ) is observed. Here too, ENDF/B-VII provides a better agreement between the measured and modeled spectra.

Figure 6 shows the difference between the gamma ray spectra for formation iron alone.

There is a major discrepancy above $5 \mathrm{MeV}$, in the total energy spectrum. In ENDF/B-VII, the doublet at 7.6 MeV and the corresponding escape peaks [6] are clearly visible. Even though the peak at $5.9 \mathrm{MeV}$ cannot be clearly identified, the associated escape peaks are clearly visible.

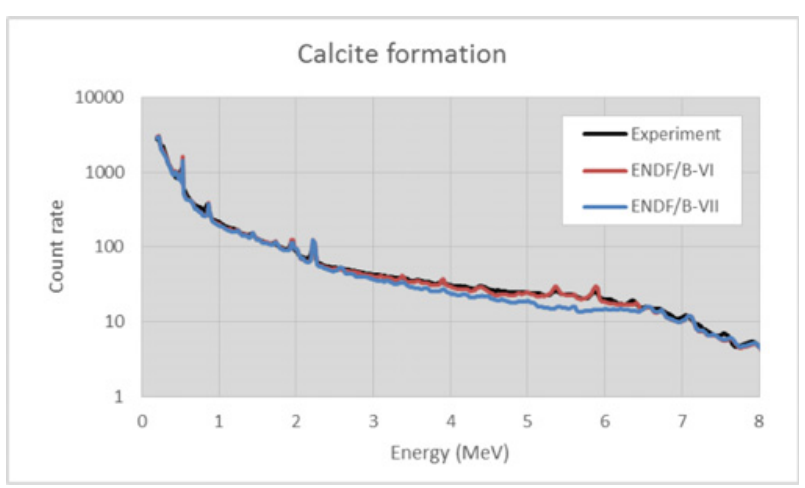

Figure 7. Comparison of ENDF/B-VI and ENDF/B-VII simulated total gamma ray spectra for a porous calcite formation.

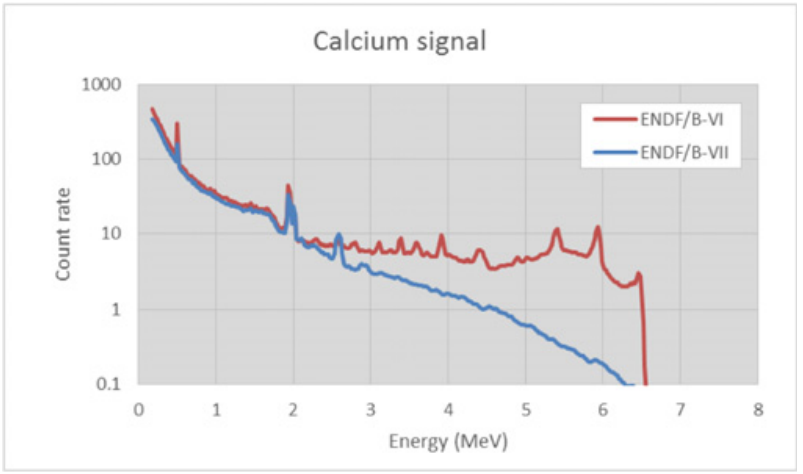

Figure 8. Comparison between ENDF/B-VI and ENDF/B-VII simulated calcium spectra in a porous formation.

This confirms that for gamma-ray production from iron, the ENDF/B-VII cross sections are the better choice.

\subsection{Calcium}

In ENDF/B-VI, calcium cross sections are only available for the natural element, not the individual isotopes. In ENDF/B-VII, cross sections are available for each isotope. Oilfield operations, generally deal with natural elements.

For calcium, we focus on the energy spectrum, because the calcium contribution to the thermal neutron absorption cross section is small compared with that of iron. Figure 7 shows the gamma ray energy spectrum that is obtained by modeling the response in a medium porosity (about $15 \%$ porosity) calcite formation.

A significant discrepancy is observed in the spectrum between 3 and $6.5 \mathrm{MeV}$. In this case, there is much better agreement with ENDF/B-VI, indicating issues with the calcium cross sections in the newer data base.

Figure 8 shows the difference between the gamma ray spectra emitted due only to the calcium atoms in the formation. The major discrepancy starts around $2.5 \mathrm{MeV}$ and ends at $6 \mathrm{MeV}$, in agreement with the observations for the total energy spectrum.

Calcium-40 has two prompt high-energy capture gamma rays at 4.4 and $6.4 \mathrm{MeV}$. Both are clearly visible along with their respective escape peaks in the modeling with ENDF/B-VI. However, they are completely absent in ENDF/B-VII modeling. Moreover, the latter simulation shows an unexplained $2.5 \mathrm{MeV}$ peak. This confirms that for gamma-ray production from calcium, the ENDF/B-VI cross section is preferred. 


\section{Cross sections needing improvement}

For several elements, the oilfield industry is interested in improved cross section data. Lanthanum, barium, bromine and cerium completely lack evaluated gamma ray production cross sections. These elements are often present in the borehole mud $(\mathrm{Ba})$ and in some gamma ray detectors ( $\mathrm{La}, \mathrm{Br}$ and $\mathrm{Ce}$ ).

Magnesium, sulfur and titanium gamma ray production cross sections have a binning that is too coarse for our spectroscopy applications.

In other studies, discrepancies have been observed in the simulated gamma ray spectra of the following elements: boron, phosphorus, zinc, molybdenum, samarium and tungsten.

\section{Conclusions}

The biggest challenge when designing nuclear tools for oil exploration using nuclear modeling is the coupled neutrongamma ray problem. In particular, neutron induced gamma ray cross sections currently do not have the same quality as the cross sections used when modeling only neutron or gamma ray transport.

As demonstrated, ENDF/B-VII is not always an improvement compared with its predecessor for simulating gamma ray production effects. This is the case in particular, when using natural compounds and their composite isotopic cross sections.

The oil industry has cross section needs for elements that are not necessarily of interest for applications in other nuclear industries. This includes the need for cross sections that are missing from the libraries or improvements to existing cross sections of elements present in downhole tools (Ti, Fe, $\mathrm{Zn}, \mathrm{Mo}$, and $\mathrm{W}$ ), the detector ( $\mathrm{La}, \mathrm{Br}, \mathrm{Ce}$, $\mathrm{Cd}, \mathrm{Sr}, \mathrm{Ge})$, the formation $(\mathrm{Mg}, \mathrm{Ca})$ and the mud $(\mathrm{Ba})$.

The authors would like to acknowledge fruitful discussions with J. Miles and R.J. Radtke concerning cross sections that need improvement.

\section{References}

[1] M.L. Mauborgne et al., "Exploring for Oil with Nuclear Physics", in International Conference on Nuclear Data for Sciences and technology, 11-16 September, Bruges, Belgium (2016)

[2] MCNPX User manual, Version 2.6.0, Los Alamos National Laboratory report LA-CP-07-1473 (2008)

[3] M. Chadwick, et al., "ENDF/B-VII.1: Nuclear Data for Science and Technology: Cross Sections, Covariances, Fission Product Yields and Decay Data", Nucl. Data Sheets 112, 2887 (2011)

[4] P.F. Rose (editor), ENDF-201: ENDF/B-VI Summary, Tech. Rep. BNL-NCS-17541 (1991)

[5] M.L. Mauborgne, et al., "Advances in LWD multiple depth of investigation array Sigma measurements", SPWLA 54th Annual Logging Symposium, San Antonio, TX, III (2013)

[6] R.B. Firestone, et al., "Database of prompt gamma rays from slow neutron capture for elemental analysis," Lawrence Berkeley National Laboratory (2004) 